\title{
Failure to Convert Rhythm
}

National Cancer Institute

\section{Source}

National Cancer Institute. Failure to Convert Rhythm. NCI Thesaurus. Code C63190.

Failure of the device therapy or set of therapies to terminate the harmful cardiac rhythm that the therapy is meant to terminate. 